\title{
Porcupine Inhibitor CGX1321
}

National Cancer Institute

\section{Source}

National Cancer Institute. Porcupine Inhibitor CGX1321. NCI Thesaurus. Code C146853.

An orally bioavailable inhibitor of the membrane-bound O-acyltransferase (MBOAT) porcupine (PORCN), with potential antineoplastic, protective and regenerative activities. Upon oral administration, PORCN inhibitor CGX1321 specifically targets and binds to PORCN in the endoplasmic reticulum (ER), thereby inhibiting the post-translational palmitoylation and secretion of Wnt ligands, thus preventing the activation of Wntmediated signaling, and inhibiting cell growth in Wnt-driven tumors. In addition, by inhibiting the secretion of Wnt ligands and preventing Wnt-mediated signaling, CGX1321 may also limit fibrosis and promote regeneration of certain tissues upon cell injury. PORCN catalyzes the palmitoylation of Wnt ligands, and plays a key role in Wnt ligand secretion. Wnt signaling is dysregulated in a variety of cancers and plays a key role in tumor cell proliferation. It also plays a key role in tissue regeneration. 